\title{
Datação de Sedimentos Pós-Barreiras no Norte do Brasil: implicações paleogeográficas
}

\author{
Sonia H. Tatumi ${ }^{1}$, Luis P. da Silva ${ }^{1}$, Elcio L. Pires ${ }^{1}$, Dilce F. Rossetti' ${ }^{2}$,Ana M. Góes ${ }^{3}$ \& \\ Casimiro S. Munita ${ }^{4}$
}

\begin{abstract}
Resumo A Formação Barreiras no nordeste do estado do Pará é recoberta discordantemente por uma variedade de estratos oxidados, de coloração vermelha clara a amarela pálida, cujas idades são apresentadas, pela primeira vez, no presente trabalho. Utilizando-se métodos de datação por luminescência opticamente estimulada (LOE), seguindo-se os protocolos MAR (Multiple Aliquot Regeneration) e SAR (Single Aliquot Regeneration) e, quando possível, ${ }^{14} \mathrm{C}$, pode-se constatar que a formação desses estratos ocorreu no final do Pleistoceno tardio ao Holoceno, tendo-se registrado idades LOE/MAR entre $430.000( \pm 60.000)$ e $3.400( \pm 400)$ anos AP. A comparação dos resultados LOE/MAR e LOE/SAR mostrou boa equivalência dos valores nas idades mais jovens, e diferenças significativas para as idades relativamente mais antigas. O fato dos estratos com idades mais antigas mostrarem evidências de deposição sob condições subaquosas levou a concluir que as diferenças obtidas indicam, muito possivelmente, que os grãos analisados não foram zerados com respeito à radiação solar no momento da deposição, refletindo a radiação adquirida em sítios deposicionais anteriores a sua última deposição. Considerando-se as idades obtidas, e integrando-se informações oriundas de publicações anteriores, conclui-se que os Sedimentos Pós-Barreiras registram a margem de um amplo paleovale, cujo registro principal ocorre a oeste da área de estudo, onde estratos correlatos são melhor desenvolvidos.
\end{abstract}

Palavras-chave: Quaternário, Sedimentos Pós-Barreiras, datação, luminescência opticamente estimulada, Ilha do Marajó.

\begin{abstract}
Dating Post-Barreiras Sediments in Northern Brazil: palaeogeographic implications. The Barreiras Formation exposed in the northeastern of the State of Pará, overlain by a variety of oxidized, light red to pale yellow strata, whose ages are presented, for the first time, in the present work. Based on dating methods applying optically stimulated luminescence (OSL) following the protocols MAR (Multiple Aliquot Regeneration) and SAR (Single Aliquot Regeneration) and, whenever possible, ${ }^{14} \mathrm{C}$, it was possible to conclude that the formation of these strata took place at the end of late Pleistocene to Holocene, with recorded LOE/MAR ages ranging from $430,000( \pm 60,000)$ to $3,400( \pm 400) \mathrm{yr}$ BP. A comparison of OSL/MAR and OSL/SAR results showed good equivalence among values for the deposits with younger ages, and significant differences for deposits with relatively older ages. The fact that strata with older ages show evidence for deposition under sub-aqueous conditions led to conclude that the differences obtained herein is more likely due to the cosmic radiation acquired during deposition in previous depositional sites. Taking into account the obtained ages, and integrating these data with information derived from previous publications, it can be concluded that the Post-Barreiras Sediments record deposition at the margin of a wide paleovalley, which display its main sedimentary record to the west of the study area.
\end{abstract}

Keywords: Quaternary, Post-Barreiras Sediments, dating, optically stimulated luminescence, Marajó Island.

INTRODUÇÃO Depósitos sedimentares formados a partir do Oligo-Mioceno possuem ampla distribuição no nordeste do Pará e litoral do Maranhão, onde estão expostos em inúmeras falésias costeiras, minas a céu aberto, barrancos de rios e cortes de estradas. Estes estratos são, em geral, delgados (em média até $40 \mathrm{~m}$ de espessura), ocorrendo em discordância erosiva sobre rochas cretáceas do Grupo Itapecuru. Várias publicações (ver referências em Rossetti \& Góes 2004) têm contribuído para o detalhamento da sucessão miocena, correspondente às formações litoestratigráficas Pirabas e Barreiras. Apesar de localmente expressivos e relevantes para a reconstituição de eventos quaternários no norte do Brasil, depósitos sobrepostos a essas unidades são, ainda, insuficientemente documentados. Em parte, isto se deve ao fato de, por muito tempo, esses estratos terem sido considerados, não como uma unidade sedimentar à parte, mas como resultado de manto intempérico sobre rochas

1 - Faculdade de Tecnologia de São Paulo - FATEC, São Paulo (SP), Brasil. E-mail: tatumi@fatecsp.br

2 - Instituto Nacional de Pesquisas Espaciais - INPE, São José dos Campos (SP), Brasil. E-mail: rossetti@dsr.inpe.br

3 - Programa de Pós-Graduação em Geoquímica e Geotectônical Geologia Sedimentar e Ambiental Universidade de São Paulo-USP, Instituto de Geociências, São Paulo (SP), Brasil. E-mail: goes@igc.usp.br

4 - Instituto de Pesquisas Energéticas Nucleares - IPEN-CNEN/SP, São Paulo (SP), Brasil. E-mail: camunita@ipen.br 
cretáceas e terciárias (Mabesone et al. 1972, Mabesone 1984). Essa visão tradicional vem sendo progressivamente modificada nas últimas décadas, graças principalmente ao reconhecimento de discordância erosiva em sua base. Esta é marcada, seja por paleossolo laterítico, seja por depósitos residuais formados por clastos resultantes do retrabalhamento deste, misturados com seixos de quartzo (Rossetti 2004). Assim, tem-se utilizado o termo informal Sedimentos Pós-Barreiras para se referenciar genericamente à sucessão estratigráfica depositada sobre essa discordância (Sá 1969, Góes 1981, Rossetti et al. 1989, 2004, Rossetti 2004).

Não há trabalhos prévios documentando a idade dos Sedimentos Pós-Barreiras expostos em superfície. A inexistência desses dados é principalmente devida à sua composição arenosa e natureza fortemente intemperizada, que dificulta a obtenção de datações radiogênicas confiáveis. Uma única exceção foi a datação radiogênica de conchas em um sambaqui que ocorre associado aos Sedimentos Pós-Barreiras na localidade de São João de Pirabas, nordeste do Pará, cuja idade foi de $5.500{ }^{14} \mathrm{C}$ anos A.P. (Francisco et al 1971). Depósitos quaternários dessa idade, e mais antigos, estão sendo recentemente bem documentados em sub-superfície na Ilha do Marajó, onde eles têm auxiliado na caracterização de um paleovale tectônico, cuja morfologia tipicamente afunilada, ainda preservada na paisagem atual, é passível de reconhecimento até as proximidades da cidade de $\mathrm{Tu}-$ curuí (Rossetti \& Valeriano 2007). Um tema relevante para a complementação desses estudos é a verificação da continuidade dos estratos relacionados ao preenchimento desse paleovale ao longo de sua margem leste, onde estes se acham bem preservados. Depósitos superficiais nessas áreas marginais ao paleovale, bem com nos seus interflúvios, são descritos informalmente como Sedimentos Pós-Barreiras. Uma hipótese a ser testada é se os Sedimentos Pós-Barreiras são correlacionáveis com estratos representativos do preenchimento do paleovale. Somente estudos sistemáticos visando a datação dos Sedimentos Pós-Barreiras poderão fornecer informações suficientes para se verificar esta suposição.

Este trabalho objetiva documentar resultados de datações dos Sedimentos Pós-Barreiras expostos ao longo da margem leste e das áreas de interflúvio, do paleovale quaternário (Fig. 1). Para isto, foram realizadas análises de luminescência opticamente estimulada (LOE), como alternativa ao método de datação por radiocarbono, em virtude da natureza intemperizada e arenosa, com baixo conteúdo orgânico, dos sedimentos. Além disto, datações LOE têm um maior alcance cronológico, permitindo datar sedimentos com idades bem mais antigas do que as obtidas por datação ${ }^{14} \mathrm{C}$. Este fato, aliado aos resultados positivos obtidos com a datação de depósitos arenosos em outras áreas do território brasileiro (Yee et al. 2003, Tatumi et al. 2002, Carneiro Filho et al. 2003) motivou o presente estudo. A determinação da idade dos Sedimentos Pós-Barreiras contribuirá para analisar sua correlação com os depósitos representativos do paleovale quaternário formado a partir de Tucuruí e ao longo da Ilha do Marajó, e conse- quentemente, melhor entender seu significado geológico e paleogeográfico.

CONTEXTO GEOLÓGICO A área de estudo, localizada no nordeste do estado do Pará, ocorre em diferentes contextos estruturais, incluindo o Sistema de Graben do Marajó (Azevedo 1991, Galvão 1991, Villegas 1994), bem como as plataformas Bragantina e do Pará (Fig. 1). O Sistema de Graben do Marajó distribuise em uma área de até $1.5 \times 10^{6} \mathrm{~km}^{2}$, consiste em uma estrutura definida por falhas orientadas para NW-SE e NE-SW, além de falhas E-W a ENE-WSW e NE-SW, que foram reativadas a partir de estruturas antigas do embasamento pré-cambriano e cretáceo (Azevedo 1991, Villegas 1994, Costa \& Hasui 1997). Este Sistema de Graben é constituído por três sub-bacias, i.e., Cametá, Limoeiro e Mexiana, formadas ao longo da Margem Equatorial Norte Brasileira, como resultado do processo de abertura do Oceano Atlântico, no início do Cretáceo. As plataformas Bragantina e do Pará correspondem às áreas do embasamento que permaneceram relativamente estáveis entre as bacias sedimentares que compõem o Sistema de Graben do Marajó, ao oeste, a Bacia de Bragança-Viseu, ao leste, a Bacia do Pará-Maranhão, ao norte, e as bacias do Amazonas e Grajaú, ao sul.

Em sub-superfície, o Sistema de Graben do Marajó (Fig. 2) é constituído por depósitos albo-cenomanianos da Formação Breves, cretáceos superiores da Formação Limoeiro, paleocenos/miocenos médios da Formação Marajó e miocenos a pleistocenos da Formação Tucunaré e Pirarucu (Galvão 1991). Na superfície, bem como em áreas das plataformas do Pará e Bragantina, depósitos correlatos, se presentes, são conhecidos como Formação Ipixuna (Neocretáceo?-Paleógeno; Rossetti 2004, Rossetti \& Santos Jr. 2006), Formação Pirabas (Oligo-Mioceno; Petri 1954, 1957, Ferreira et al. 1984, Góes et al. 1990), Formação Barreiras (Meso/ Neomioceno; Rossetti 2000, 2004, Rossetti \& Góes 2004) e Sedimentos Pós-Barreiras (Quaternário; Rossetti et al. 1989, Rossetti 2004).

Mais do que representando uma unidade estratigráfica única, o mapeamento estratigráfico em afloramentos indica que os Sedimentos Pós-Barreiras no nordeste do Pará e litoral do Maranhão incorporam, pelo menos, dois episódios de sedimentação, referenciados informalmente de Sedimentos Pós-Barreiras I e II (Rossetti 2004). Esta subdivisão foi confirmada em subsuperfície pela utilização de radar de penetração no solo (Rossetti \& Góes 2001, Rossetti et al. 2001). Os Sedimentos Pós-Barreiras I, inferiores, formam um pacote de até $10 \mathrm{~m}$ de espessura, que consiste, predominantemente, em areias de coloração vermelho-clara a alaranjada, friáveis a endurecidas, maciças, bioturbadas, de seleção moderada a boa, granulometrias em geral finas a médias, podendo ser localmente grossas a conglomeráticas. Pelitos e argilitos bioturbados são localmente presentes. Esses depósitos são recobertos, em discordância erosiva, pelos Sedimentos Pós-Barreiras II, que representam um pacote de espessuras variáveis, com médias em torno de 2 a $5 \mathrm{~m}$, composto por areias 


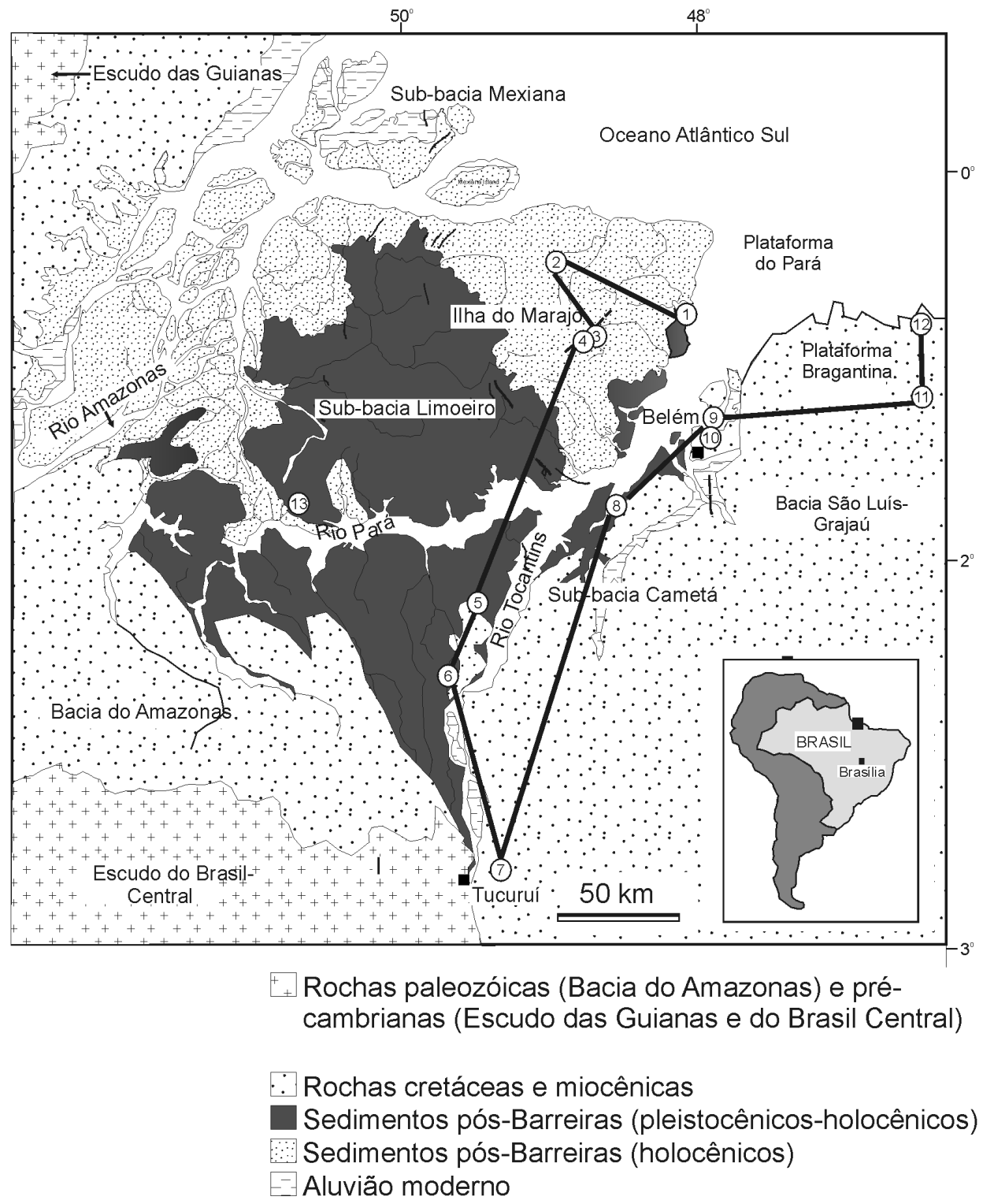

Figura 1 - Mapa geológico simplificado, com a localização da área de estudo no nordeste do estado do Pará e dos perfis litoestratigráficos (1 a 12) que forneceram as amostras datadas neste estudo. Observar a morfologia triangular dos Sedimentos Pós-Barreiras relacionada com o preenchimento de um paleovale, que se inicia na cidade de Tucuruí e amplifica para NNW em direção à Ilha do Marajó.

finas a muito finas, bem selecionadas, com fragmentos de carvão dispersos e, eventualmente, fragmentos de cerâmica. As cores variam desde tipicamente amarela clara a amarela dourada, laranja acinzentada, marrom amarelado e amarelo amarronzado. $\mathrm{O}$ acamamento maciço é a estrutura mais comum, porém ocorrem, também, estruturas de dissipação de dunas sugestivas de contribuição eólica (Rossetti et al. 1989).

MATERIAIS E MÉTODOS Este estudo foi baseado na análise de 32 amostras, coletadas ao longo de 12 perfis litoestratigráficos, representativos dos Sedimentos Pós-Barreiras expostos no nordeste do estado do Pará. As datações foram obtidas por luminescência opticamente estimulada (LOE, 25 amostras), utilizan- do-se protocolo MAR (Multiple Aliquot Regeneration). Cinco amostras de idades MAR, antigas e jovens, foram selecionadas para serem analisadas também pelo protocolo SAR (Single Aliquot Regeneration). Embora mais oneroso em termos de custos e tempo, relativamente ao protocolo MAR, este procedimento vem sendo, mais recentemente, apontado como de maior precisão na datação de depósitos sedimentares (Buykaert et al. 2008, Bassinet et al. 2006, Murray et al. 2007, Lomax et al. 2007). Portanto, a análise de algumas amostras pelos dois protocolos visou comparação dos resultados, a fim de testar se a metodologia MAR na área de estudo resulta em dados confiáveis. Adicionalmente, quando possível, fez-se análises por radiocarbono ${ }^{14} \mathrm{C}(7$ amostras). 


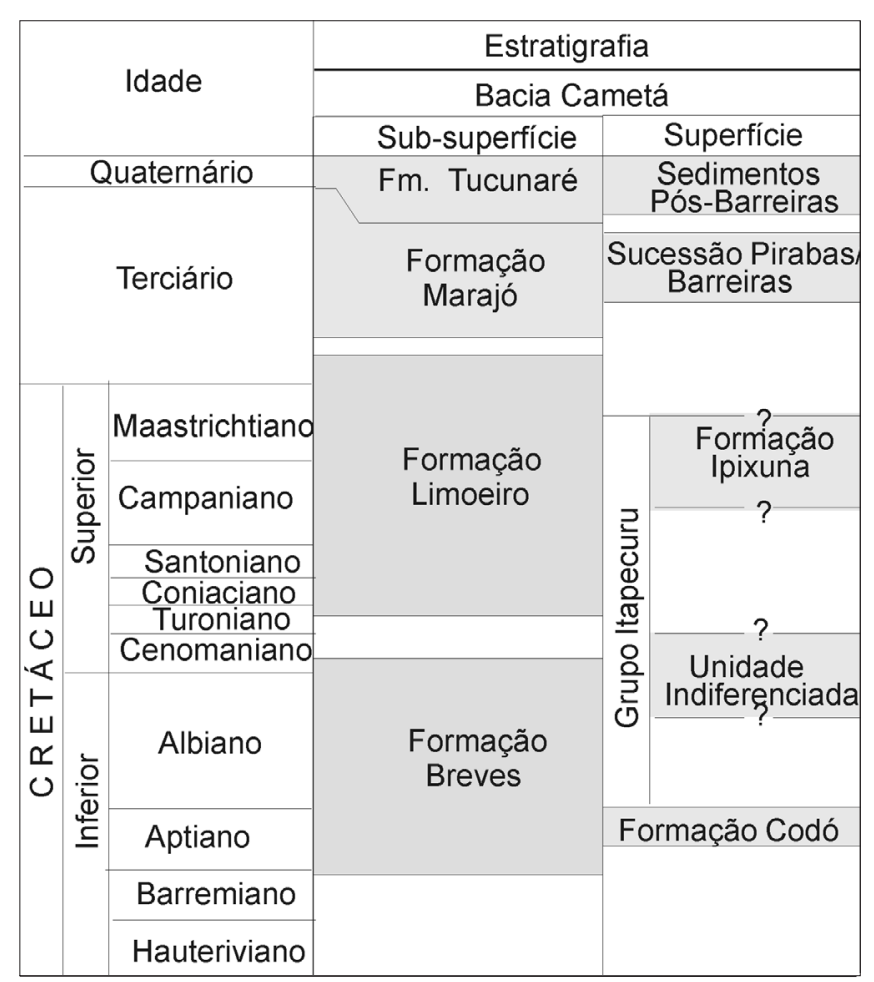

Figura 2 - Coluna litoestratigráfica simplificada da área de estudo, com a designação das unidades em sub-superficie e superficie.

Os métodos de datação por luminescência de cristais baseiam-se na interação da radiação ionizante (radiação- $\gamma$, partícula- $\beta$ e partícula- $\alpha$ ) com o cristal natural. Quando a radiação incide sobre o cristal, ocorre a ionização do mesmo, com a criação de pares de cargas positivas e negativas no seu interior. Estas cargas podem ser aprisionadas em armadilhas, formadas por defeitos e impurezas da rede cristalina, constituindo, assim, estados meta-estáveis, que podem ter um tempo de vida estimada em milhões de anos. Quando o cristal é aquecido, há a liberação das cargas, que podem ser recombinadas por meio da emissão de luz, fenômeno denominado termoluminescência (TL). Se o cristal for estimulado opticamente, ele pode emitir luz em comprimento de onda diferente daquele usado na estimulação, sendo a luz emitida desta forma denominada luminescência opticamente estimulada (LOE). Quanto maior o tempo de submissão do cristal às radiações ionizantes, emitidas pelos radioisótopos naturais ${ }^{238} \mathrm{U},{ }^{235} \mathrm{U},{ }^{232} \mathrm{Th} \mathrm{e}$ $\left.{ }^{40} \mathrm{~K}\right)$ que estão no solo, ou pela radiação cósmica, maior será a concentração de estados meta-estáveis no cristal e, conseqüentemente, maior será a intensidade de emissão TL ou LOE. A Idade (I) da amostra depende da dose acumulada $(D A)$ no cristal, em decorrência da exposição do mesmo à radiação ionizante e da taxa $(T)$ de radiação incidente no cristal, por ano. Assim a equação da idade da amostra pode ser expressa como (Eq. 1):

A taxa $T$ é determinada pela concentração dos isótopos naturais em cada amostra, os quais podem ser convertidos em taxa de dose através de tabela citada em

$$
I=\frac{D A(G y)}{T_{\gamma}+T_{\beta}+T_{\alpha}+T_{\text {raioscósmicos }}(G y / \text { ano })}
$$

Aitken (1998). A contribuição da partícula- $\alpha$ é, geralmente, negligenciada, pois sua ionização atua apenas na superfície dos grãos, podendo ser retirada com ácido fluorídrico. A DA pode ser determinada calibrando-se os cristais, isto é, descobrindo-se a relação entre a intensidade de luz em função da dose acumulada no cristal, por meio da utilização do método da regeneração total. Quando submetido a doses conhecidas de radiação por meio de uma fonte radioativa calibrada (fonte de ${ }^{60} \mathrm{Co}$ ), o cristal é "zerado", traçando-se, assim, curvas de calibração. O cálculo da Paleodose $(P)$ é feito com base no ponto onde a área média (alíquotas múltiplas) da LOE natural intercepta a reta de crescimento.

No presente trabalho, foi utilizado o método de análise por ativação com nêutrons instrumental (AANI) para determinar a concentração de U, Th e K. Para isto, as amostras foram irradiadas junto com o padrão NISTSRM-1633b no reator IEA-R1m do IPEN-CNEN/SP, sob um fluxo de nêutrons térmicos de $5 \times 10^{12} \mathrm{n} \mathrm{cm}^{-2} \mathrm{~s}^{-1} \mathrm{du}-$ rante 1 h. O espectro dos raios- $\gamma$ foi obtido após 7 e 25 dias de decaimento com um detector de Ge-hiperpuro, modelo GX 1925 da Canberra com placa MCA S-100 de 8192 canais e resolução de $1.9 \mathrm{keV}$ no pico de 1332,49 $\mathrm{keV}$ do ${ }^{60} \mathrm{Co}$. A taxa da radiação cósmica de $237 \pm 6 \mu \mathrm{Gy} /$ ano foi determinada a partir de medidas dos espectros da radiação- $\gamma$ feitas no Rio Guamá, Belém ( $\mathrm{S} \mathrm{01}{ }^{\circ}$ 28,894'; W $\left.048^{\circ} 27,207^{\prime}\right)$, com detector de $\mathrm{NaI}(\mathrm{Tl})$ da Canberra Inc.

No protocolo SAR, foram analisadas cerca de 20 alíquotas com massa de $10 \mathrm{mg}$ aproximadamente para cada amostra, cujas curvas de calibração foram obtidas seguindo-se protocolo de Murray \& Wintle (2000). Uma dose teste foi efetuada para corrigir perdas de sensibilidade na resposta do sinal de LOE, devido ao uso contínuo de uma mesma alíquota. A diferença desse protocolo para o MAR é que, neste último caso, cada dose regenerativa é feita com uma alíquota diferente. $\mathrm{O}$ protocolo SAR tem a vantagem de fornecer valores das idades para o estudo cronológico dos eventos, além de informações a respeito da flutuação desses valores em um mesmo depósito. Os dados de LOE foram obtidos com o sistema de leitura automática de TL/LOE da Daybreak Nuclear Instruments Inc, Modelo 1100.

As análises de radiocarbono foram obtidas somente in locu, onde é possível coletar fragmentos de carvão ou sedimentos pouco intemperizados que ainda apresentassem algum conteúdo de matéria orgânica. As amostras foram analisadas no Beta Analytic Radiocarbon Dating Laboratory, utilizando-se datação radiométrica padrão após passarem por pré-tratamento com ácidos, visando remoção de eventuais carbonatos e quebra de compostos orgânicos. As idades das amostras foram calibradas de acordo com calendário anual utilizandose o programa de procedimentos Pretoria, baseado em dados de anéis de crescimento de árvores, como curvas de calibração (Talma \& Vogel 1993). Os dados apresentados correspondem a idades convencionais, expressos 
em anos antes do presente (anos AP).

DESCRIÇÃO DOS PERFIS E IDADES Os perfis estudados e os resultados das datações obtidas são apresentados nas figuras 3 e 4 e tabelas 1 e 2 . Em geral, os Sedimentos Pós-Barreiras expostos na área de estudo são delgados, limitando-se a, no máximo, $10 \mathrm{~m}$ de espessura. O perfil 1 é representativo de uma falésia lateralmente contínua por várias dezenas de metros que ocorre na cidade de Salvaterra, em frente ao Hotel Pousada dos Aruãs. Nesta localidade, os Sedimentos Pós-Barreiras, de até $3 \mathrm{~m}$ de espessura, são bem representados no topo do perfil (Fig. 4A,B). Estes consistem, na base, em argilitos plano-paralelos seguidos de argilitos e arenitos de cores variegadas, típicos da sucessão Pirabas/Barreiras. Como característico em muitas de suas áreas de ocorrência (ver Rossetti 2001, 2004), nesta localidade, esta sucessão é recoberta por uma superfície de descontinuidade de relevo erosivo, marcada por concreções ferruginosas verticalizadas desenvolvidas sobre horizonte caulinítico, representativo de paleossolo laterítico. Acima desse horizonte, ocorre delgada camada de arenito, que é recoberta por outra superfície de descontinuidade, também com relevo erosivo e salientada por depósitos residuais constituídos de fragmentos de concreções ferruginosas. Os Sedimentos Pós-Barreiras ocorrem sobrejacentes a esta superfície, sendo representados por um pacote de $3 \mathrm{~m}$ de areias endurecidas, de coloração amarela, quartzosas, maciças, granulometria fina e bem selecionada, contendo grânulos de quartzo e fragmentos de carvão dispersos. Três datações LOE/MAR foram obtidas neste pacote, que indicam deposição entre $32.000( \pm 3.000)$ e 3.400 $( \pm 4.000)$ anos AP, além de uma idade radiocarbono de $15.180 \pm 240$ anos AP.

O perfil 2 corresponde a uma trincheira ao sul da Fazenda Apii. Neste local ocorrem argilitos cinzas a esverdeados na base, com laminação plano-paralela incipiente, sobrepostos por areias amarelas, que grada para cima a areias brancas, de granulometria fina e bem selecionada. Duas idades foram obtidas neste local, incluindo-se LOE/MAR $4.200( \pm 500)$, superior, e ${ }^{14} \mathrm{C}$ 3.500 anos AP, inferior.

Os perfis 3 e 4 foram obtidos em trincheiras nas proximidades da cidade de Cachoeira do Arari, e apresentam os Sedimentos Pós-Barreiras com espessuras inferiores a $1 \mathrm{~m}$, sendo compostos por areias friáveis, brancas, quartzosas, finas, bem selecionadas e maciças. No perfil 3, estes estratos recobrem uma superfície de descontinuidade contendo concreções ferruginosas similares às observadas no perfil 1. Esta superfície desenvolve-se sobre depósitos areno-argilosos, de coloração variegada (vermelha, violácea, branco-amarelada), maciços e extremamente bioturbados, relacionados à Formação Barreiras. É interessante notar que duas amostras oriundas desta unidade basal destinadas à datação revelaram-se saturadas, possivelmente por terem ultrapassado o limite do método de 1 milhão de anos. No perfil 4, os Sedimentos Pós-Barreiras consistem em duas camadas de poucos centímetros de espessura, sen- do uma inferior, representada por areia friável, marrom escura, maciça, bem selecionada e extremamente bioturbada, com as seguintes idades, da base para o topo: LOE/MAR 430.000 ( \pm 60.000) e LOE/SAR $191.000( \pm$ 27.000); LOE/MAR 112.000 ( \pm 14.000$)$ e LOE/SAR 76.000 ( \pm 8.000); e LOE/MAR $7.700( \pm 900)$ e LOE/ SAR $12.000( \pm 2.300)$ anos AP.

Os perfis 5 e 6 correspondem a cortes de estrada, situados a poucos quilômetros a oeste da cidade de Cametá, onde os Sedimentos Pós-Barreiras possuem pouco mais de $2 \mathrm{~m}$ de espessura, sendo constituídos de areias com características similares aos depósitos correlatos do perfil 1. Como naquele perfil, os Sedimentos Pós-Barreiras no perfil 5 são também sobrepostos à superfície de descontinuidade com relevo erosivo e marcada por depósitos residuais compostos por clastos de laterita ferruginosa. Três análises LOE/MAR foram feitas nesses depósitos. Para as duas amostras inferiores, houve consistência nos resultados de $22.000( \pm 2.000)$ e $7.500( \pm 900)$ anos AP, enquanto que a amostra mais superior, representativa de uma camada de areia branca, friável, de granulometria fina que ocorre no topo das areias amarelas endurecidas, indicou idade de 209.000( \pm 28.000$)$ anos AP. O perfil 6 apresenta Sedimentos Pós-Barreiras de idade de ${ }^{14} \mathrm{C} 7.790 \pm 130 \mathrm{AP}$.

No perfil 7, resultante de uma grande escavação para retirada de areias nas proximidades da cidade de Breu Branco, os Sedimentos Pós-Barreiras, com $10 \mathrm{~m}$ de espessura, ocorrem sobrepostos discordantemente a conglomerados arcoseanos relacionados à Formação Ipixuna, de idade cretácea superior a paleógena. Neste perfil, a metade inferior dos Sedimentos Pós-Barreiras é litologicamente similar ao perfil 1, tendo apresentado idade LOE/MAR=21.900 ( \pm 3.000$)$ anos AP. Já a metade superior do perfil é constituída por areias de cor marrom e, no topo, brancas, ambas friáveis, maciças, bem selecionadas e contendo abundantes fragmentos de carvão dispersos. Nestes últimos depósitos, obtiveram-se idades LOE/MAR=6.800 ( \pm 1.200$)$ anos AP e, acima desta, duas idades radiocarbono de $8.810 \pm 60$ anos AP (inferior) e 115,59 $\pm 0,48$ anos AP (superior).

O perfil 8 corresponde a uma falésia com cerca de $10 \mathrm{~m}$ de altura, descrita nas proximidades da cidade de Abaetetuba (Fig. 4C,D), em cuja base ocorrem argilitos mosqueados, maciços, de topo marcado por superfície de descontinuidade lateralmente contínua e contendo paleossolo com raízes abundantes. Estes depósitos, relacionados à sucessão Pirabas/Barreiras inferior, são sobrepostos por arenitos de cor vermelha, bem selecionados, maciços e marcados, no topo, por superfície de descontinuidade, também lateralmente contínua, de relevo erosivo e salientada por concreções ferruginosas in situ. Os Sedimentos Pós-Barreiras ocorrem diretamente sobre esta superfície, sendo representados na base por pacote granodecrescente consistindo em conglomerados formados por seixos de quartzo, que gradam para cima a arenitos brancos, endurecidos, maciços, e de granulometria variando de grossa a fina. Análise de uma amostra deste intervalo arenoso indicou idade LOE/MAR=34.000 ( \pm 4.000) anos AP. No topo 


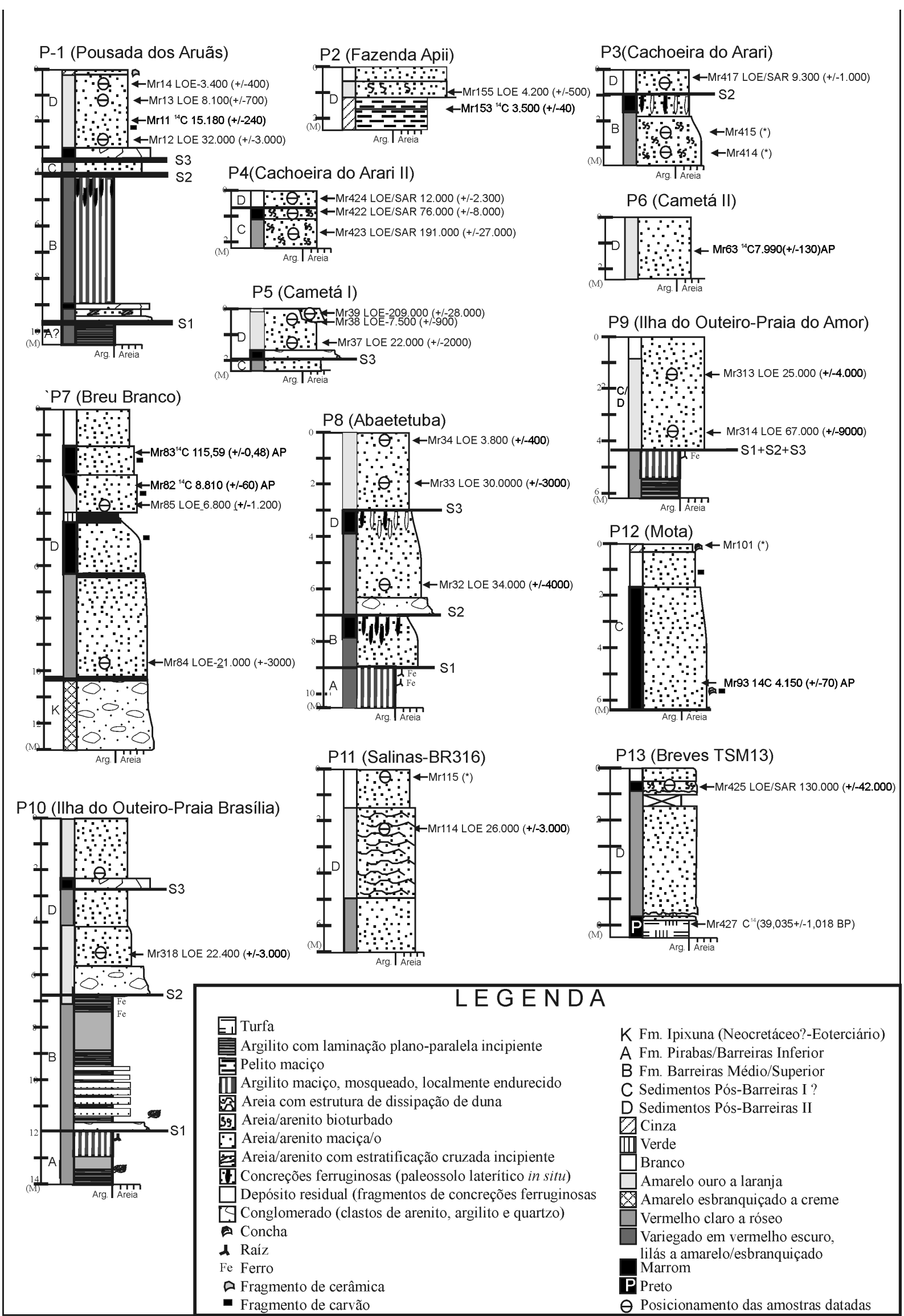

Figura 3 - Perfis litoestratigráficos representativos dos Sedimentos Pós-Barreiras dispostos ao longo da margem leste do paleovale quaternário, bem como áreas de interflúvio. As amostras e as idades $T L, L O E$ e de radiocarbono ${ }^{14} \mathrm{C}$, acham-se posicionadas à direita dos perfis (ver figura 1 para localização dos perfis; *=amostras saturadas). 

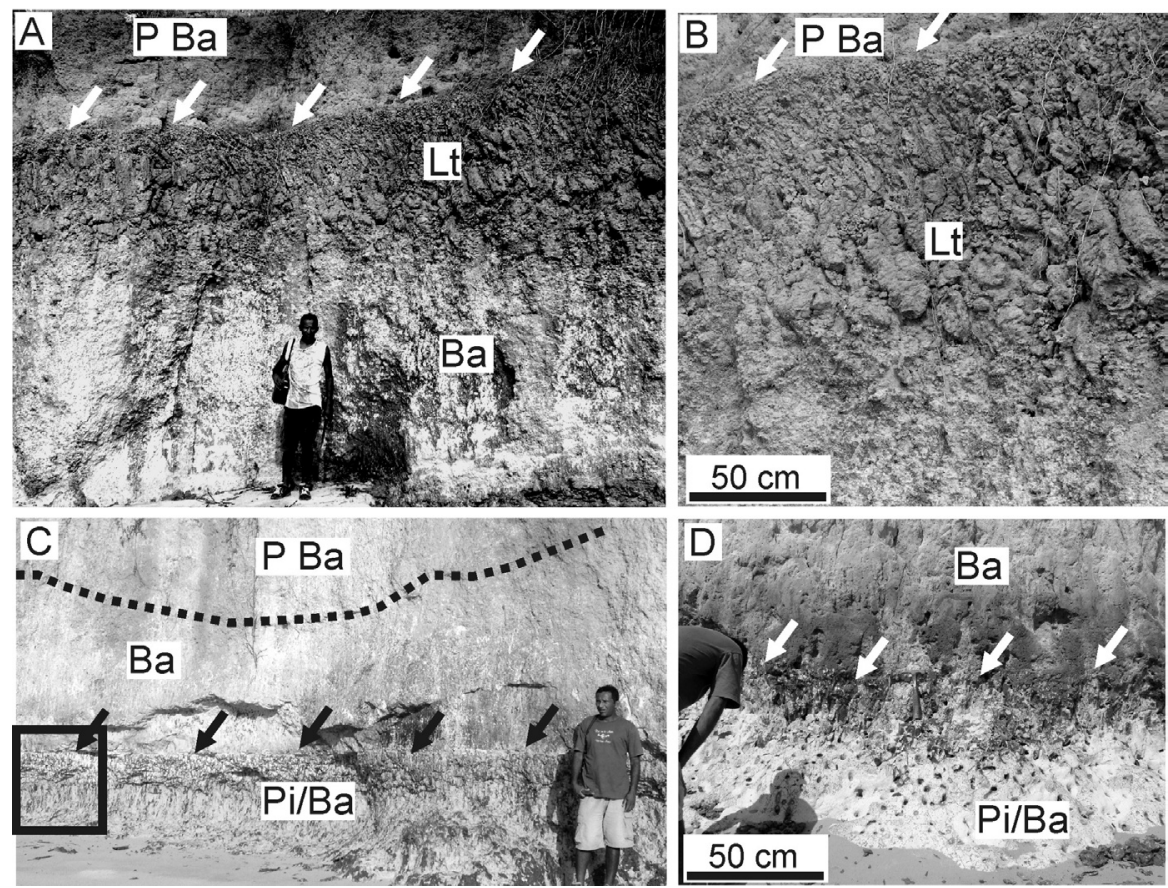

Figura 4 - Exemplos de afloramentos ilustrando os depósitos estudados. A) Vista geral do perfil 1 (Praia dos Aruãs), onde os Sedimentos Pós-Barreiras ocorrem discordantemente sobre superficie de descontinuidade (setas pretas) marcada por erosão e paleossolo laterítico, este caracterizado, no topo, por concreções ferruginosas. Este paleossolo acha-se desenvolvido sobre a Formação Barreiras. B) Detalhe da superficie de descontinuidade vista na figura A. C) Perfil 8 (Abaetetuba), ilustrando a sucessão Pirabas/Barreiras inferior sendo sobreposta por superficie de descontinuidade (setas), salientada por abundantes marcas de raizes. Acima desta, ocorre a Formação Barreiras Média/Superior, bastante intemperizada, que é sobreposta discordantemente (linha pontilhada) pelos Sedimentos Pós-Barreiras. Concreções lateríticas estão ausentes neste local, mas elas aparecem lateralmente no perfil. D) Detalhe da figura C, ilustrando a superficie de descontinuidade (setas) no topo da Formação Pirabas/Barreiras superior, marcada por abundantes marcas de raizes.

da sucessão granodecrescente, observa-se superfície de descontinuidade marcada por ferruginização incipiente. Sobre esta, ocorre um pacote de 2,5 $\mathrm{m}$ de areia similar aos Sedimentos Pós-Barreiras do perfil 1, onde foram obtidas as seguintes idades LOE/MAR, da base para o topo: $30.400( \pm 3.000)$ e $3.800( \pm 400)$ anos AP.

Os perfis 9 e 10 foram descritos ao longo de falésias na Ilha do Outeiro, sendo o primeiro na Praia do Amor (Fig. 5A) e o segundo na Praia Brasília (Fig. 5B,C). A sucessão Pirabas/Barreiras é bem representada na porção inferior e média desses perfis, sendo constituída por intercalações de arenitos, argilitos e depósitos heterolíticos, localmente bem estruturados. Nesses depósitos, ocorre superfície de descontinuidade marcada por paleossolo com abundantes raízes, que é correlacionável àquela observada no perfil 8 . Em ambos os perfis, a sucessão Pirabas/Barreiras é recoberta por Sedimentos Pós-Barreiras. Na Praia do Amor (perfil 9), esses chegam a $4 \mathrm{~m}$ de espessura, sendo representados por areias amarelas similares àquelas do perfil 1, com idades LOE/ MAR de $67.000( \pm 9.000)$ (inferior) e $25.000( \pm 4.000)$ anos AP (superior). Na Praia Brasília (perfil 10), ocorre uma sucessão mais espessa de cerca de aproximadamente $7 \mathrm{~m}$, composta de conglomerado intraformacional, arenito litificado, vermelho, maciço na base, que é sobreposta discordantemente por areias amarelas similares às da Praia do Amor, com idade LOE/MAR= $37.000( \pm$ 5.000) anos AP.

O perfil 11 corresponde a um afloramento em corte de estrada ao longo da PA-324, que liga Capanema a Salinópolis. Nessa localidade, os Sedimentos Pós-Barreiras possuem $7 \mathrm{~m}$ de espessura, sendo representados por areias levemente endurecidas, amareloalaranjadas a levemente avermelhadas na base, bem selecionadas e bem arrendadas, maciças ou com estruturas de dissipação de dunas, de onde se obteve uma idade LOE/MAR=26.000 $( \pm 3.000)$ anos AP. Para o topo, ocorre um pacote de areia branca, maciça, friável, bem selecionada, cuja amostra mostrou-se saturada.

O perfil 12, na localidade do Mota, município de Salinópolis, mostra uma sucessão de areias vermelhas claras a amarelas, de granulometrias bem selecionadas e arredondadas, maciças na base e com estruturas de dissipação de dunas no topo. Estes depósitos contém 

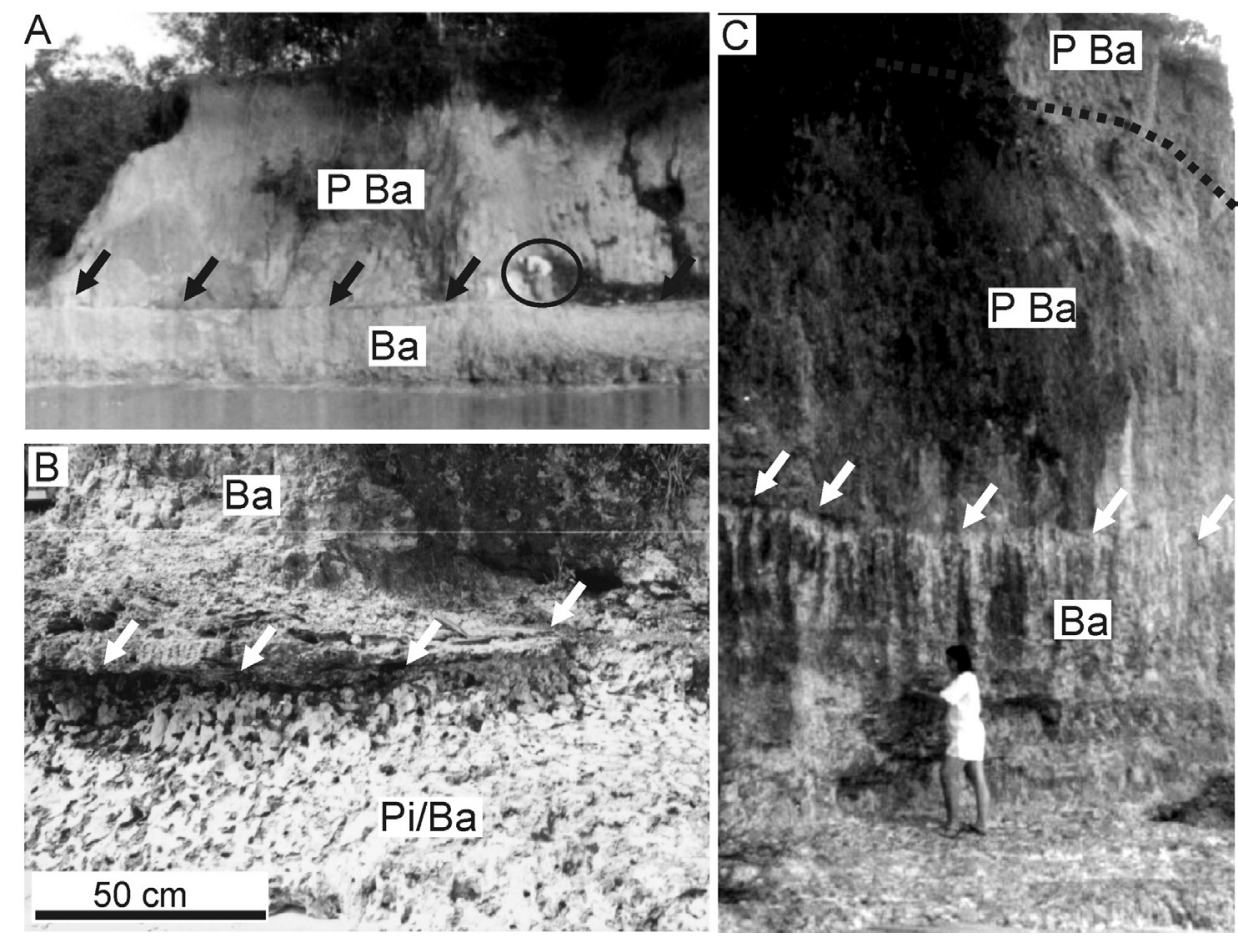

Figura 5 - A) Perfil 9 (Ilha do Outeiro-Praia do Amor), ilustrando a Formação Barreiras sobreposta por Sedimentos Pós-Barreiras em discordância erosiva (setas) (círculo indica pessoa para escala); B) Detalhe da superficie de descontinuidade ilustrada em A na Praia Brasilia. Notar que esta superficie é marcada por paleossolo com abundantes marcas de raizes (comparar com a figura 4C); C) Perfil 10 (Ilha do Outeiro-Praia Brasilia), ilustrando a Formação Barreiras sobreposta discordantemente (setas) por Sedimentos Pós-Barreiras. Notar a presença de descontinuidade dentro destes (linha pontilhada) (Pi/Ba=Formação Pirabas/Barreiras Inferior; Ba=Formação Barreiras Média/Superior; PBa=Sedimentos Pós-Barreiras; $L t=$ horizonte de paleossolo laterítico).

fragmentos de carvão com idade ${ }^{14} \mathrm{C}$ de $4.145( \pm 70)$ anos AP, porém a amostra analisada por LOE/MAR mostrou-se saturada.

Um último perfil 13, obtido nas proximidades da cidade de Breves, foi inserido neste estudo. Apesar de localizado à margem oeste do paleovale, este perfil foi adicionado pela similaridade que mostra com os perfis 3 e 4. Isto é, nessa localidade, ocorre também depósito de areia marrom caracterizado por abundantes bioturbações, a menos de $1 \mathrm{~m}$ de profundidade, cuja idade LOE/MAR foi de 333.300 anos AP, com idades LOE/SAR correspondente de $130.000( \pm 42.000)$ anos AP. Este dado é inconsistente com idade mais jovem (i.e., de apenas ${ }^{14} \mathrm{C} 39.035 \pm 1.018$ anos AP) obtida acerca de $6 \mathrm{~m}$ de profundidade.

DISCUSSÕES Antes de se proceder com uma discussão geológica sobre o significado dos dados analisados, é importante ressaltar que os resultados das datações LOE possuem, em geral, grande consistência, tanto ao longo de um mesmo perfil, quanto entre as localidades analisadas, revelando uma ampla faixa de idades para os Sedimentos Pós-Barreiras. As datações radiogênicas serviram de complementação às datações por LOE, uma vez que estas se mostraram muito próximas dos valores obtidos através da LOE. Os experimentos LOE/SAR realizados com algumas amostras selecionadas (i.e., amostras MR417, MR422, MR423, MR424 e MR425), revelaram que, para as idades mais jovens, os valores foram bastante próximos dos obtidos por LOE/MAR. Isto indica que as datações LOE/SAR obtidas em depósitos da área de estudo com idades de até poucas dezenas de milhares de anos mostram-se precisas, podendo ser utilizadas em interpretações geológicas.

Entretanto, para idades maiores, os valores LOE/SAR mostraram diferenças de, até, metade dos valores LOE/MAR. Exceto pela amostra MR314, todas as demais amostras com idades antigas (i.e., amostras MR 39, MR422, MR 423 e MR 425), que chegam a algumas centenas de milhares de anos, provém sistematicamente de areias finas, de coloração branca ou marrom, cuja característica comum é a abundância de bioturbações. Essas amostras foram excluídas das interpretações geológicas abaixo, uma vez que, dado o elevado grau de bioturbação, concluiu-se que a deposição ocorreu subaquosamente, seja em ambiente continental, seja em ambiente com influência marinha, não havendo tempo suficiente de zerar o sinal da radiação solar adquirida 
Tabela 1 - Resultados de concentrações de isótopos radioativos ( $\left.U={ }^{235} U+{ }^{238} U{ }^{232} \mathrm{Th} e{ }^{40} \mathrm{~K}\right)$, taxas de dose anual (DA), paleodoses $(P)$ e idades $\left(I_{L O E}\right)$ de sedimentos da Ilha do Marajó obtidas a partir de análises de Luminescência Opticamente Estimulada utilizando-se os protocolos MAR (Multiple Aliquot Regeneration) e SAR (Single Aliquot Regeneration).

\begin{tabular}{|c|c|c|c|c|c|c|c|c|}
\hline $\begin{array}{c}\text { Amos- } \\
\text { Tra }\end{array}$ & $\mathrm{U}(\mathrm{ppm})$ & ${ }^{232} \mathrm{Th}$ (ppm) & ${ }^{40} \mathrm{~K}(\mathrm{mg} / \mathrm{g})$ & $\begin{array}{l}\mathrm{DA}(\mathrm{mGy} / \\
\text { ano) }\end{array}$ & $\begin{array}{c}\text { P(Gy) } \\
\text { LOE-MAR }\end{array}$ & $\begin{array}{c}\text { P(Gy) } \\
\text { LOE-SAR }\end{array}$ & $\begin{array}{c}\mathrm{I}_{\mathrm{LOE}} \\
\mathrm{MAR} \\
\text { (kanos) }\end{array}$ & $\begin{array}{c}\mathrm{I}_{\mathrm{LOE}} \\
\text { SAR (kanos) }\end{array}$ \\
\hline MR12 & $4,4 \pm 0,4$ & $17,5 \pm 1,0$ & - & $2,62 \pm 0,18$ & $85 \pm 2$ & & $32 \pm 3$ & \\
\hline MR13 & $4,9 \pm 0,4$ & $21,3 \pm 1,1$ & - & $3,04 \pm 0,18$ & $24,5 \pm 0,6$ & & $8,1 \pm 0,7$ & \\
\hline MR14 & $4,3 \pm 0,5$ & $14,7 \pm 0,8$ & - & $2,39 \pm 0,19$ & $8,2 \pm 0,3$ & & $3,4 \pm 0,4$ & \\
\hline MR32 & $3,2 \pm 0,5$ & $17,3 \pm 1,0$ & $3,2 \pm 0,1$ & $2,30 \pm 0,20$ & $77 \pm 3$ & & $34 \pm 4$ & \\
\hline MR33 & $4,2 \pm 0,4$ & $22,1 \pm 1,2$ & - & $2,91 \pm 0,19$ & $86 \pm 4$ & & $30 \pm 3$ & \\
\hline MR34 & $4,8 \pm 0,4$ & $21,9 \pm 1,2$ & - & $3,05 \pm 0,19$ & $12,4 \pm 0,4$ & & $3,8 \pm 0,4$ & \\
\hline MR37 & $2,9 \pm 0,3$ & $18,5 \pm 1,0$ & - & $2,31 \pm 0,15$ & $52 \pm 2$ & & $22 \pm 2$ & \\
\hline MR38 & $3.8 \pm 0,5$ & $16,1 \pm 0,9$ & - & $2,36 \pm 0,20$ & $17,8 \pm 0,6$ & & $7,5 \pm 0,9$ & \\
\hline MR39 & $0,53 \pm 0,10$ & $0,30 \pm 0,03$ & - & $0,43 \pm 0,33$ & $90 \pm 5$ & & $209 \pm 28$ & \\
\hline MR84 & $1,6 \pm 0,3$ & $7,6 \pm 0,4$ & - & $1,16 \pm 0,11$ & $24,5 \pm 0,7$ & & $21 \pm 3$ & \\
\hline MR85 & $1,2 \pm 0,3$ & $6,0 \pm 0,4$ & - & $0,93 \pm 0,11$ & $6,3 \pm 0,4$ & & $6,8 \pm 1,2$ & \\
\hline MR101 & \multicolumn{8}{|c|}{ Amostra saturada } \\
\hline MR114 & $0,9 \pm 0,2$ & $3,2 \pm 0,2$ & - & $0,65 \pm 0,07$ & $16,8 \pm 0,5$ & & $26 \pm 3$ & \\
\hline MR115 & \multicolumn{8}{|c|}{ Amostra saturada } \\
\hline MR313 & $1,4 \pm 0,3$ & $5,9 \pm 0,4$ & - & $0,98 \pm 0,11$ & $24,8 \pm 0,7$ & & $25 \pm 4$ & \\
\hline MR314 & $1,2 \pm 0,2$ & $4,5 \pm 0,2$ & $0,01 \pm 0,01$ & $0,82 \pm 0,07$ & $55 \pm 3$ & & $67 \pm 9$ & \\
\hline MR319 & $2,57 \pm 0,04$ & $10,7 \pm 0,5$ & $0,44 \pm 0,01$ & $1,64 \pm 0,48$ & $60 \pm 7$ & & $37 \pm 5$ & \\
\hline MR155 & $1,3 \pm 0,1$ & $4,2 \pm 0,2$ & $0,37 \pm 0,01$ & $0,78 \pm 0,05$ & $3,3 \pm 0,2$ & & $4,2 \pm 0,5$ & \\
\hline MR414 & \multicolumn{8}{|c|}{ Amostra saturada } \\
\hline MR415 & \multicolumn{8}{|c|}{ Amostra saturada } \\
\hline MR417 & $1,8 \pm 0,2$ & $6,5 \pm 0,3$ & $0,57 \pm 0,02$ & $1,12 \pm 0,06$ & $9,4 \pm 0,4$ & $10,4 \pm 0,6$ & $8,4 \pm 0,7$ & $9,3 \pm 1,0$ \\
\hline MR422 & $0,36 \pm 0,09$ & $1,69 \pm 0,08$ & $0,105 \pm 0,003$ & $0,39 \pm 0,03$ & $44 \pm 2$ & $30 \pm 1$ & $112 \pm 14$ & $76 \pm 8$ \\
\hline MR423 & $1,8 \pm 0,3$ & $6,8 \pm 0,3$ & $0,9 \pm 0,1$ & $1,15 \pm 0,11$ & $495 \pm 20$ & $22 \pm 1$ & $430 \pm 60$ & $191 \pm 27$ \\
\hline MR424 & $0,94 \pm 0,02$ & $0,72 \pm 0,03$ & $0,39 \pm 0,01$ & $0,47 \pm 0,28$ & $3,6 \pm 0,2$ & $5,6 \pm 0,5$ & $7,7 \pm 0,9$ & $12,0 \pm 2,3$ \\
\hline MR425 & $0,7 \pm 0,6$ & $1,86 \pm 0,09$ & $0,45 \pm 0,01$ & $0,50 \pm 0,03$ & $170 \pm 1$ & $66 \pm 10$ & $337 \pm 24$ & $130 \pm 42$ \\
\hline
\end{tabular}

Tabela 2 - Resultados das datações obtidas por radiocarbono ${ }^{14} \mathrm{C}$.

\begin{tabular}{c|c|c|c}
\hline $\begin{array}{c}\text { Perfil/ } \\
\text { Amostra }\end{array}$ & Tipo de Material & ${ }^{14} \mathrm{C}$ anos AP & Idade calibrada AP/calibração 2 sigma \\
\hline P1/MR-11 & carvão & $15.180+/-240$ & $19.000-17.360$ \\
\hline P6/MR63 & carvão & $7.990+/-130$ & $9.260-8.460$ \\
\hline P7/MR82 & carvão & $8.810+/-60$ & $10.150-9.600$ \\
\hline P7/MR 83 & carvão & $115,59+/-0,48$ & $4.850-4.500$ e 4.480-4.440 \\
\hline P2/MR153 & sedimento orgânico & $3.500+/-40$ & $3.870-3.670$ \\
\hline P12/MR93 & sedimento orgânico & $4.150(+/-70)$ & $4.850-4.500$ e $4.480-4.440$ \\
\hline P13/MR427 & sedimento orgânico & $39,035+/-1.018$ & - \\
\hline
\end{tabular}

no ambiente deposicional anterior. Com isto, as idades documentadas refletem o tempo de deposição dos sedimentos em sítios deposicionais anteriores. Depósitos similares de areia marrom, contendo abundantes bioturbações, foram também verificados na região de Breves, onde se obteve idades correlatas de várias centenas de milhares de anos, como ilustrado no perfil 12. Estudo sedimentológico realizado naquela localidade suporta que estes depósitos foram formados subaquosamente, possivelmente em ambiente com influência marinha 
(Rossetti \& Góes 2008).

Considerando-se as demais idades, pode-se concluir que os Sedimentos Pós-Barreiras que ocorrem expostos diretamente à superfície, ou em trincheiras, na margem do paleovale quaternário mapeado na Ilha do Marajó, foram depositados no final do período Pleistoceno ao Holoceno. É interessante notar que depósitos com idade similares, de até 40.000 anos AP, são registrados no interior do paleovale a várias dezenas de metros de profundidade, chegando, excepcionalmente, a $120 \mathrm{~m}$ de profundidade (Rossetti et al. 2008). Esta situação estratigráfica leva a supor que os Sedimentos Pós-Barreiras são muito mais espessos do que tradicionalmente registrados em superfície, particularmente ao longo da margem oeste do paleovale quaternário. As pequenas espessuras desses depósitos em superfície, onde eles são limitados, na base, pela Formação Barreiras ou, mais raramente, pela Formação Ipixuna, é condizente com deposição em áreas marginais do paleovale. Nestas localidades, a taxa de sedimentação é menor devido ao reduzido espaço de acomodação. Em direção ao interior do paleovale, cuja origem acha-se relacionada a reativações tectônicas, como discutido anteriormente, ocorrem áreas favoráveis a apresentar maior espaço de acomodação, devido ao deslocamento do terreno por falhas. Nessas áreas, a taxa de sedimentação pode ser significativamente maior que nas áreas de interflúvios, gerando depósitos sedimentares mais espessos.

Apesar de ter sido constatada, nos Sedimentos Pós-Barreiras, superfície de descontinuidade interna associada com lateritização incipiente ou depósitos residuais formados por clastos oriundos de horizontes de paleossolos lateríticos, considerando-se as datações LOE aqui apresentadas, observa-se que esta superfície separa estratos de idades próximas (ver perfis P8 e P10), representando curto hiato na sedimentação.

A ocorrência de depósitos na margem leste do paleovale contendo idades quaternárias similares às registradas dentro do paleovale, aponta haver correspondência estratigráfica entre esses estratos. Portanto, os Sedimentos Pós-Barreiras representam a expressão superficial da Formação Pirarucu, caracterizando, portanto, a deposição marginal do paleovale descrito na área de estudo. Reativações de zonas de falhas pré-existentes na região levaram ao estabelecimento de um amplo paleovale, que se estende desde a cidade de Tucuruí até a Ilha do Marajó, no sentido NNW (Rossetti \& Valeriano 2007). Durante o período Pleistoceno tardio ao Holoceno, essa área foi preenchida com sedimentos, processo este mais intenso em sítios favoráveis à maior subsidência, devida ao abatimento de blocos. Enquanto a parte interna do vale era preenchida, suas porções marginais também recebiam sedimentos, porém em menor volume, já que estes se constituíam em locais relativamente estáveis e, portanto, com menos chance de formação de espaço para acomodação de sedimentos. Os sedimentos formados nas áreas marginais ao paleovale, além de serem de espessuras reduzidas, estiveram sujeitos, por mais tempo, a condições de erosão, intemperismo e pedogênese. Estes processos resultaram em depósitos homogêneos e pobres em matéria orgânica, como é típico dos Sedimentos Pós-Barreiras. Estes depósitos contrastam com sedimentos correlatos, porém bem estruturados e ricos em matéria orgânica que ocorrem no interior do paleovale (Rossetti et al. 2008, Miranda \& Rossetti 2007).

CONCLUSÕES A utilização do método de datação por LOE/MAR possibilitou, pela primeira vez, uma estimativa da idade dos Sedimentos Pós-Barreiras que ocorrem no nordeste do estado do Pará. Com base nos dados apresentados neste trabalho, pode-se concluir que a deposição dessa unidade geológica ocorreu no final do Pleistoceno ao Holoceno. A correspondência das idades obtidas na área de estudo com dados de sub-superfície registrados oriundos da porção central do paleovale definido anteriormente entre a região entre Tucuruí e a Ilha do Marajó, leva a concluir que os Sedimentos Pós-Barreiras representam a deposição marginal desse paleovale. A natureza delgada e a forte influência de processos subaéreos dos Sedimentos Pós-Barreiras, são consistentes com essa interpretação.

A comparação dos resultados LOE/MAR e LOE/SAR revelou boa correspondência de idades para os sedimentos formados na ordem de poucas dezenas de milhares de anos. Portanto, o protocolo LOE/MAR, que envolve procedimento mais simplificado relativamente ao LOE/SAR, pode ser utilizado para a datação dos Sedimentos Pós-Barreiras na área de estudo. Para depósitos mais antigos, isto é, de algumas centenas de milhares de anos, as diferenças entre os dois protocolos foram bastante significativas. Isto, no entanto, parece estar ligado muito mais à presença de sedimentos depositados em condições subaquosas, do que a problemas reais da aplicação dos protocolos LOE. Estudos futuros são, ainda, necessários a fim de detectar depósitos formados em condições subaéreas com idades antigas similares, que possam ser utilizados para melhor testar a precisão do protocolo LOE/MAR na datação de depósitos quaternários da área de estudo.

Agradecimentos Este trabalho é uma contribuição ao Projeto FAPESP 04/15518-6.

\section{Referências}

Aitken M.J. 1998. An Introduction to Optical Dating. London, Oxford University Press, 267p.

Azevedo RP. 1991. Tectonic evolution of Brazilian Equatorial Continental Margin Basins. Tese de Doutoramento,
University of London, London, 412p.

Bassinet C., Mercier N., Miallier D., Pilleyre T., Sanzelle S., Valladas H. 2006.Thermolumenscence of heated quartz grains: Intercomparisons between SAR and multiple-ali- 
quot additive dose techniques. Radiation Measurements, 41:803-808.

Buylaert J.P., Murray A.S., Vandenberghe D., Vriend M., DE Corte F., Van den haute P. 2008. Optical dating of Chinese loess using sand-sized quartz:Establishing a time frame for Late Pleistocene climate changes in the western part of the Chinese loess plateau. Quaternary Geochronology, 3:99-113.

Carneiro Filho A., Tatumi S.H., Yee M. 2003. Dunas Fósseis na Amazônia. Ciência Hoje-SBPC, 191:24-29.

Costa J.B.S. \& Hasui Y. 1997. Evolução geológica da Amazônia. In: Costa M.L \& Angélica R.S. (eds.) Contribuições à Geologia da Amazônia. SBG, Belém, p. 15-19.

Ferreira C.S., Vicalvi M.A., Macedo A.C.M. 1984. Os limites meridionais da Bacia de Pirabas. In: SBG, Congresso Brasileiro de Geologia, 33, Anais, 1:326-329.

Francisco B.H.R., Loewenstein P., Silva G.G. 1971. Contribuição à geologia da Folha de São Luís (SA-23), no Estado do Pará. Bol. Mus. Paraense Emílio Goeldi, Serie Geol., 17:1-40.

Galvão M.V.G. 1991. Evolução Termodinâmica da Bacia do Marajó, Estado do Pará, Brasil. Dissertação de Mestrado, Universidade de Ouro Preto, 193p.

Góes A.M. 1981. Estudo sedimentológico dos sedimentos Barreiras, Ipixuna e Itapecuru, no nordeste do Estado do Pará e noroeste do Maranhão. Dissertação de Mestrado, Universidade Federal do Pará, Belém, 55p.

Góes A.M., Rossetti D.F., Nogueira A.C.R., Toledo P.M. 1990. Modelo deposicional preliminar da Formação Pirabas no nordeste do Estado do Pará. Bol. Mus. Paraense Emílio Goeldi, Serie Ciênc. Terra, 2:3-15.

Lomax J.,Hilgers A., Twidale C.R., Bourne J.A., Radtke U. 2007. Treatment of broad palaeodose distributions in OSL dating of dune sands from the western Murray Basin,south Australia. Quaternary Geochronology, 2:51-56.

Mabesoone J.M., Campos Silva A., Buerlen K. 1972. Estratigrafia e origem do Grupo Barreiras em Pernambuco, Paraíba e Rio Grande do Norte. Revista Brasileira de Geologia, 2:173-188.

Mabesoone J.M. 1984. Cenozóico nordestino: sistemas e seqüências deposicionais. In: SBG, Simp. Geol., Nordeste, 11, Atas, p. 142-150.

Miranda M.C. \& Rossetti D.F. 2007. Paleoambientes do Pleistoceno tardio-Holoceno na área do Lago Arari, Ilha do Marajó. In: ABEQUA, 11, Bol. Resumos, CD-Rom.

Murray A.S. \& Wintle A.G. 2000. Luminescence dating of quartz using an improved single aliquot regenerativedose protocol. Radiation Measurements, 32:57-73.

Petri S. 1954. Foraminíferos fósseis da Bacia do Marajó. Bol. Fac. Fil. Ciênc.Letras da USP, Geol., 134:1-172.

Petri S. 1957. Foraminíferos miocênicos da Formação Pirabas. Bol. Fac. Fil. Ciênc. Letras USP, Geol., 216:1-79.

Rossetti D.F. 2000. Influence of low amplitude/high frequency relative sea-level changes in a wave-dominated estuary (Miocene), São Luís Basin, northern Brazil. Sed. Geol., 133:295-324.

Rossetti D.F. 2004. Paleosurfaces from northeastern Ama- zonia as a key for reconstructing paleolandscapes and understanding weathering products. Sed. Geol., 169:51174.

Rossetti D.F. 2006. Evolução sedimentar miocênica nos Estados do Pará e Maranhão. Geol. USP. Ser. Cient.,6:7-18.

Rossetti D.F. \& Góes A.M. 2001. Imaging Upper Tertiary to Quaternary deposits from northern Brazil applying ground penetrating radar. Rev. Bras. Geoc., 31:195-202.

Rossetti D.F. \& Góes A.M. 2004. Geologia. In D.F. Rossetti \& A.M. Góes (eds.) O Neógeno da Amazônia Oriental Belém, Museu Paraense Emílio Goeldi, p. 13-52.

Rossetti D.F. \& Góes A.M. 2008. Late Quaternary drainage dynamics in northern Brazil based on the study of a large paleochannel from southwestern Marajó Island. Anais da Academia Brasileira de Ciências, 80:579-593.

Rossetti D.F. \& Santos Jr. A.E.A. 2004 . Facies architecture in a tectonically-influenced estuarine incised valley fill of Miocene age, northern Brazil. J. South American Earth Sci., 17:267-284.

Rossetti D.F. \& Santos Jr. A.E.A. 2006. Analysing the origin of the Upper Cretaceous-?Lower Tertiary Rio Capim semi-flint (Pará State, Brazil) under a sedimentologic perspective. Sed. Geol., 186:133-144.

Rossetti D.F. \& Valeriano M.M. 2007. Evolution of the lowest Amazon basin modeled from the integration of geological and SRTM topographic data. Catena, 70:253-265.

Rossetti D.F., Góes A.M., Souza L.S.B. 2001. Estratigrafia da sucessão sedimentar Pós-Barreiras (zona bragantina, Pará) com base em radar de penetração no solo. Rev. Bras. Geof., 19:113-130.

Rossetti D.F., Truckenbrodt W., Góes A.M. 1989. Estudo paleoambiental e estratigráfico dos sedimentos Barreiras e Pós-Barreiras na região bragantina, nordeste do Pará. Bol. Mus. Paraense Emílio Goeldi, Ser. Ciênc. Terra, 1:25-74.

Rossetti D.F., Góes A.M., Valeriano M.M., Miranda M.C.C. 2008. Quaternary tectonics in a passive margin: Marajó Island, northern Brazil. J. Quat. Sci., 23:121-135.

Sá J.H.S. 1969. Contribuição à geologia dos sedimentos terciários e quaternários da região bragantina, estado do Pará. Bol. Geol., 3:20-36.

Talma A.S. \& Vogel J.C. 1993. A simplified approach to calibrating C14 dates. Radiocarbon, 35:317-322.

Tatumi S.H., Carneiro Filho A., Kowata E.A., Yee M., Schwartz D. 2002. TL and OSL dating of eolian dunes of the Negro river Basin, Brazil. Advances in ESR applications, 18:87-191.

Villegas J.M.C. 1994. Geologia estrutural da Bacia do Marajó. Dissertação de Mestrado, Univ. Federal Pará, 119p.

Yee M., Tatumi S.H., Suguio K., Barreto A.M.F., Munita C.S., Paiva R.P. 2003. Thermoluminescence (TL) dating of inactive dunes from the Rio Grande do Norte Coast, Brasil. J. Coastal Res., 35:293-299

Manuscrito ID11062 Submetido em 02 de abril de 2008 Aceito em 20 de setembro de 2008 Sistema eletrônico de submissão 